To Appear in the Astronomical Journal

Preprint typeset using $\mathrm{LAT}_{\mathrm{E}} \mathrm{X}$ style emulateapj v. 5/2/11

\title{
THE TYPE IIB SUPERNOVA 2013DF AND ITS COOL SUPERGIANT PROGENITOR
}

\author{
Schuyler D. Van DyK ${ }^{1}$, WeiKang Zheng ${ }^{2}$, Ori D. Fox ${ }^{2}$, S. Bradley Cenko ${ }^{3}$, Kelsey I. ClubB ${ }^{2}$, Alexei \\ V. Filippenko ${ }^{2}$, Ryan J. Foley ${ }^{4,5}$, Adam A. Miller ${ }^{6,7}$, Nathan Smith ${ }^{8}$, Patrick L. Kelly ${ }^{2}$, William H. LeE ${ }^{9}$, Sagi \\ Ben-Ami ${ }^{10}$, AND Avishay GaL-YAM ${ }^{10}$ \\ To Appear in the Astronomical Journal
}

\begin{abstract}
We have obtained early-time photometry and spectroscopy of Supernova (SN) 2013df in NGC 4414. The SN is clearly of Type IIb, with notable similarities to SN 1993J. From its luminosity at secondary maximum light, it appears that less ${ }^{56} \mathrm{Ni}\left(\lesssim 0.06 M_{\odot}\right)$ was synthesized in the SN 2013df explosion than was the case for the SNe IIb 1993J, 2008ax, and 2011dh. Based on a comparison of the light curves, the SN 2013df progenitor must have been more extended in radius prior to explosion than the progenitor of SN 1993J. The total extinction for SN 2013df is estimated to be $A_{V}=0.30$ mag. The metallicity at the SN location is likely to be solar. We have conducted Hubble Space Telescope (HST) Target of Opportunity observations of the SN with the Wide Field Camera 3, and from a precise comparison of these new observations to archival HST observations of the host galaxy obtained 14 years prior to explosion, we have identified the progenitor of SN 2013df to be a yellow supergiant, somewhat hotter than a red supergiant progenitor for a normal Type II-Plateau SN. From its observed spectral energy distribution, assuming that the light is dominated by one star, the progenitor had effective temperature $T_{\text {eff }}=4250 \pm 100 \mathrm{~K}$ and a bolometric luminosity $L_{\text {bol }}=10^{4.94 \pm 0.06} L_{\odot}$. This leads to an effective radius $R_{\text {eff }}=545 \pm 65 R_{\odot}$. The star likely had an initial mass in the range of $13-17$ $M_{\odot}$; however, if it was a member of an interacting binary system, detailed modeling of the system is required to estimate this mass more accurately. The progenitor star of SN 2013df appears to have been relatively similar to the progenitor of SN 1993J.
\end{abstract}

Subject headings: galaxies: individual (NGC 4414) — stars: evolution — supernovae: general supernovae: individual (SN 2013df)

\section{INTRODUCTION}

A core-collapse supernova (SN) is the final stage of evolution for stars with initial mass $M_{\text {ini }} \gtrsim 8 M_{\odot}$ (e.g., Woosley et al. 2002). The compact remnant of this explosion is thought to be either a neutron star or a black hole. Stars which reach the terminus of their evolution with most of their hydrogen-rich envelope intact - single red supergiants (RSGs) - produce Type II (specifically Type II-Plateau) supernovae (SNe). As the envelope is stripped away, either through vigorous mass loss or via mass transfer in a binary system, the results are the hydrogen-free, yet helium-rich, Type Ib SNe and the hydrogen-free and helium-poor (or helium-free) Type Ic SNe; see Filippenko (1997) for a review of SN classifica-

\footnotetext{
${ }^{1}$ Spitzer Science Center/Caltech, Mail Code 220-6, Pasadena, CA 91125; email: vandyk@ipac.caltech.edu.

2 Department of Astronomy, University of California, Berkeley, CA 94720-3411.

3 Astrophysics Science Division, NASA Goddard Space Flight Center, Mail Code 661, Greenbelt, MD 20771.

${ }^{4}$ Harvard-Smithsonian Center for Astrophysics, 60 Garden Street, Cambridge, MA 02138.

${ }^{5}$ Department of Astronomy, University of Illinois, UrbanaChampaign, IL 61801.

${ }^{6}$ Jet Propulsion Laboratory, MS 169-506, Pasadena, CA 91109.

${ }^{7}$ Hubble Fellow.

8 Steward Observatory, University of Arizona, Tucson, AZ 85720 .

${ }^{9}$ Instituto de Astronomía, Universidad Nacional Autónoma de México, Apdo. Postal 70-264, Cd. Universitaria, México DF 04510, México

10 Benoziyo Center for Astrophysics, The Weizmann Institute of Science, Rehovot 76100, Israel.
}

tion. Intermediate to the $\mathrm{SNe}$ II and $\mathrm{SNe} \mathrm{Ib}$ are the Type IIb, which retain a low-mass $\left(\lesssim 1 M_{\odot}\right)$ hydrogen envelope prior to explosion. SNe IIb are intrinsically rare $(\sim 10-11 \%$ of all core-collapse SNe; Smith et al. 2011; Li et al. 2011). The first identified case was SN 1987K (Filippenko 1988); well-studied examples are the nearby SN 1993J in Messier 81 (e.g., Richmond et al. 1996; Matheson et al. 2000) and SN 2011dh in Messier 51 (e.g., Arcavi et al. 2011; Ergon et al. 2013). The progenitor channel for SN 1993J which has garnered most favor over the years has been a massive interacting binary system (e.g., Podsiadlowski et al. 1993; Woosley et al. 1994; Maund et al. 2004; Stancliffe \& Eldridge 2009). A similar model has been introduced for SN 2011dh (Benvenuto et al. 2013). The progenitor is the mass donor via Rochelobe overflow.

Spectroscopically, SNe IIb exhibit at early times emission and absorption features due to $\mathrm{H}$, resembling $\mathrm{SNe}$ II, which subsequently give way to the emergence of the He I absorption lines typical of SNe Ib (e.g., Filippenko et al. 1993; Chornock et al. 2011), with broad H emission reappearing in the nebular phase (e.g., Filippenko et al. 1994; Matheson et al. 2000; Taubenberger et al. 2011; Shivvers et al. 2013). Although rarely seen owing to its very short duration early in the evolution of a SN, a rapid decline after an initial peak has been observed among SNe IIb (e.g., Richmond et al. 1994; 1996; Roming et al. 2009; Arcavi et al. 2011). This is interpreted as adiabatic cooling after the SN shock has broken out through the star's surface; the duration of this cooling is governed primarily by the radius of the progenitor (Chevalier \& Fransson 2008; Nakar \& Sari 2010; Rabinak \& Waxman 2011; Bersten et 
al. 2012). The SN light curves reach a secondary maximum, as a result of thermalization of the $\gamma$-rays and positrons emitted during the radioactive decay of ${ }^{56} \mathrm{Ni}$ and ${ }^{56} \mathrm{Co}$, followed by a smooth decline. The light-curve shapes resemble those of SNe Ib (Arcavi et al. 2012).

As the number of all core-collapse SNe having directly identified progenitor stars is very small $(\sim 20$ at the time of this writing), we have been extraordinarily fortunate that, up to this point, the progenitors of three SNe IIb have been identified, including SN 1993J (Aldering et al. 1994; Van Dyk et al. 2002; Maund et al. 2004; Maund \& Smartt 2009), SN 2008ax in NGC 4490 (Crockett et al. 2008), and SN 2011dh in Messier 51 (Maund et al. 2011; Van Dyk et al. 2011). Each of these stars exhibits distinctly different properties, although all show indications of envelope stripping prior to explosion: the SN 1993J progenitor has been characterized as a K-type supergiant, with initial mass $\sim 13-22 M_{\odot}$ (Van Dyk et al. 2002; Maund et al. 2004); for SN 2008ax it was difficult to fit a single supergiant to the observed colors ( $\mathrm{Li}$ et al. 2008; Crockett et al. 2008), and the possible initial mass range for the progenitor is large, $\sim 10-28 M_{\odot}$; and for SN 2011dh we now know that the $\sim 6000 \mathrm{~K}$ yellow supergiant identified by Maund et al. (2011) and Van Dyk et al. (2011) was indeed the star that exploded (Ergon et al. 2013; Van Dyk et al. 2013b), with initial mass $\sim 12$ $15 M_{\odot}$ (Maund et al. 2011; Murphy et al. 2011; Bersten et al. 2012). In addition, Ryder et al. (2006) may have detected at very late times the massive companion to the SN IIb 2001ig in NGC 7424 (Silverman et al. 2009). Furthermore, Chevalier \& Soderberg (2010) have divided SN IIb progenitors into those that are compact (radius $\sim 10^{11} \mathrm{~cm}$; e.g., SN 2008ax) and those that are extended ( 10 ${ }^{13} \mathrm{~cm} ;$ SN 1993J), although SN 2011dh appears to be an intermediate case (Horesh et al. 2013). Each new example provides us with an increased understanding of this SN subtype and of the massive stars which give rise to these explosions.

In this paper we consider SN 2013df in NGC 4414, shown in Figure 1. It was discovered by Ciabattari et al. (2013) on June 7.87 and 8.83 (UT dates are used throughout). Cenko et al. (2013) provided spectroscopic confirmation on June 10.8 that it is a Type II SN. The resemblance to SN 1993J at early times suggested to Cenko et al. that SN 2013df would evolve to be a SN IIb. Here we present early-time photometric and spectroscopic observations of SN 2013df, which demonstrate that it is indeed a SN IIb. In a telegram, we presented a preliminary identification of three progenitor candidates for the SN (Van Dyk et al. 2013a). We provide here a much better identification, through high-resolution imaging of the $\mathrm{SN}$, and show that none of the three sources turned out actually to be at the SN position. We will characterize the nature of the probable progenitor. The host galaxy, NGC 4414, is a nearby, isolated, flocculent spiral galaxy, with an inclination of $55^{\circ}$ (Vallejo et al. 2002). Thornley \& Mundy (1997) measured a global star formation rate of $1.3 M_{\odot} \mathrm{yr}^{-1}$, comparable to that of other Sc galaxies. SN 2013df is at a nuclear offset of $32^{\prime \prime} \mathrm{E}, 14^{\prime \prime} \mathrm{N}$, along an outer spiral arm. The galaxy was also host to the likely Type Ia SN 1974G (e.g., Schaefer 1998). We adopt the Cepheid-based distance modulus $\mu_{0}=31.10 \pm 0.05 \mathrm{mag}$ (distance $16.6 \pm 0.4 \mathrm{Mpc}$ ) established by Freedman et al.
(2001).

\section{OBSERVATIONS}

\subsection{Early-Time Photometry}

We have observed the SN using the $0.76 \mathrm{~m}$ Katzman Automatic Imaging Telescope (KAIT; Filippenko et al. 2001) at Lick Observatory in BVRI between 2013 June 13.7 and July 18.7. Point-spread function (PSF) photometry was applied using the DAOPHOT (Stetson 1987) package from the IDL Astronomy User's Library ${ }^{11}$. The instrumental magnitudes and colors of the SN were transformed to standard Johnson-Cousins BVRI using two stars in the SN field which are in the Sloan Digital Sky Survey (SDSS) catalog, and by following this prescription $^{12}$ to convert from the SDSS to the Johnson-Cousins system.

Data in rizJH were also obtained with the multichannel Reionization And Transients InfraRed camera (RATIR; Butler et al. 2012) mounted on the $1.5-\mathrm{m}$ Harold L. Johnson telescope at the Mexican Observatorio Astronómico Nacional on Sierra San Pedro Mártir in Baja California, México (Watson et al. 2012). Typical observations include a series of 60 -s exposures, with dithering between exposures. RATIR's fixed infrared (IR) filters cover half of their respective detectors, automatically providing off-target IR sky exposures while the target is observed in the neighboring filter. Master IR sky frames were created from a median stack of off-target images in each IR filter. No off-target sky frames were obtained with the optical CCDs, but the small galaxy size and sufficient dithering allowed for a sky frame to be created from a median stack of all the images in each filter. Flat-field frames consist of evening sky exposures. Given the lack of a cold shutter in RATIR's design, IR "dark" frames are not available. Laboratory testing, however, confirms that dark current is negligible in both IR detectors (Fox et al. 2012). The data were reduced, coadded, and analyzed using standard CCD and IR processing techniques in IDL and Python, utilizing online astrometry programs SExtractor (Bertin \& Arnouts 1996) and SWarp ${ }^{13}$. Calibration was performed using a single comparison star in the SN field that also has reported fluxes in both 2MASS (Skrutskie et al. 2006) and the SDSS Data Release 9 Catalogue (Ahn et al. 2012).

We did not yet possess template images of the host galaxy (i.e., prior to the SN discovery or when the SN has faded to invisibility) to subtract from the images with the SN present in each band. Consequently, the photometry presented here should be considered preliminary; however, the SN is far from the main light from the host galaxy, so results including template subtraction prior to photometry might not substantially differ from what we present here.

\subsection{Early-Time Spectroscopy}

We have obtained a number of spectra of the SN at early times, using the Kast spectrograph (Miller \&

\footnotetext{
11 http://idlastro.gsfc.nasa.gov/contents.html.

$12 \mathrm{http}$ ://www.sdss.org/dr7/algorithms/sdssUBVRITransform.html \#Lupton2005.

13 SExtractor and SWarp can be accessed from http://www.astromatic.net/software.
} 
Stone 1993) on the Lick Observatory 3-m Shane telescope and the DEep Imaging Multi-Object Spectrograph (DEIMOS; Faber et al. 2003) on the Keck-II 10-m telescope. Ultraviolet (UV) spectroscopy has also been obtained using the Hubble Space Telescope (HST) Space Telescope Imaging Spectrograph (STIS) as part of program GO-13030 (PI: A. V. Filippenko). The results of these HST observations will be presented in a future paper, together with the bulk of the ground-based optical spectra. However, here we present and analyze a representative spectrum obtained on 2013 July 11.2 with DEIMOS using the $600 \mathrm{l} \mathrm{mm}^{-1}$ grating.

\subsection{HST Imaging}

The region of the host galaxy containing the SN site was observed with the HST Wide Field Planetary Camera 2 on 1999 April 29 by program GO-8400 (PI: K. Noll), as part of the Hubble Heritage Project. The bands used were F439W (two individual images with 40 s exposure times and two with $1000 \mathrm{~s}$ ), F555W (four 400-s exposures), F606W (two 60-sec exposures), and F814W (two 40-s and four 400-s exposures). The F555W and F814W data were combined with images obtained by programs GO-5397 and GO-5972 at an earlier time for the rest of the galaxy, and drizzled into mosaics in each band at the scale 0 .'05 pix $^{-1}$ by Holwerda et al. (2005).

We have also observed the SN on 2013 July 15 with HST using the Wide Field Camera 3 (WFC3) UVIS channel in F555W, as part of our Target of Opportunity program GO-12888 (PI: S. Van Dyk). The observations consisted of 28 5-s exposures; the short exposure time was intended to avoid saturation in each frame by the bright SN.

\section{ANALYSIS \\ 3.1. Light Curves}

In Figure 2 we display the early-time KAIT and RATIR light curves in all bands for SN 2013df. We also include the very early $V$ measurement from June 11.202 by Stan Howerton ${ }^{14}$. We compare these curves with those at BVRIJH for SN 1993J (Richmond et al. 1994; 1996; Matthews et al. 2002) and for SN 2011dh (Arcavi et al. 2011; Van Dyk et al. 2013b; Ergon et al. 2013). We also compare the $z$-band light curve of SN 2008ax (Pastorello et al. 2008). The curves for the comparison SNe were adjusted in time and relative brightness to match the curves of SN 2013df, particularly at the secondary maximum in each band. Clearly, from its overall photometric similarity with the other SNe, SN 2013df appears to be a SN IIb; see Arcavi et al. (2012) for a general description of SN IIb light-curve shapes. What is most notable from the comparison is that the postshock-breakout cooling of SN 2013df occurred at a later epoch in all bands, relative to that of SN 1993J. The post-breakout decline of SN 2011dh occurred at an even earlier relative epoch (e.g., Arcavi et al. 2011; Bersten et al. 2012).

We show in Figure 3 the absolute $V$-band light curve of SN 2013df, relative to those of SNe 1993J, 2008ax, and 2011dh. (We do not make this comparison based on bolometric or pseudo-bolometric luminosity, since, as noted,

${ }^{14}$ http://www.flickr.com/photos/watchingthesky/9035874997. we consider the present photometry of SN 2013df to be preliminary.) The light curve of SN 2008ax is from Pastorello et al. (2008), rather than from Taubenberger et al. (2011), since the former has somewhat more complete coverage through the secondary maximum. The epochs of the secondary maxima in $V$ for SNe 1993J, 2008ax, and $2011 \mathrm{dh}$ are from Richmond et al. (1996), Pastorello et al. (2008), and Ergon et al. (2013), respectively. This epoch for SN 2013df was determined by comparing its light curve to that of each of the three comparison SNe. The light curves have all been adjusted for extinction and for the distances to their host galaxies (distance to SN 2013df, above; 3.6 Mpc for SN 1993J, Freedman et al. 2001; 8.4 Mpc for SN 2011dh, Van Dyk et al. 2013b; and 9.6 Mpc for SN 2008ax, Pastorello et al. 2008). We discuss below our estimate of the extinction for SN 2013df. Ergon et al. (2013) have estimated the total extinction for SN 1993J and SN 2011dh to be $A_{V} \approx 0.53$ and $A_{V} \approx 0.22$ mag, respectively, and Pastorello et al. (2008) estimate $A_{V}=0.93 \mathrm{mag}$ for SN 2008ax (all assuming a Cardelli, Clayton, \& Mathis 1989 reddening law and $R_{V}=3.1$ ).

SN 2013df appears to be the least luminous of all of these SNe. The differences in the peak luminosity likely arise from differences in the synthesized ${ }^{56} \mathrm{Ni}$ mass (e.g., Perets et al. 2010). For comparison, the ${ }^{56} \mathrm{Ni}$ produced in the comparison $\mathrm{SNe}$ was $\sim 0.07-0.15 M_{\odot}, \sim 0.10$ $0.15 M_{\odot}$, and $\sim 0.06 M_{\odot}$ in SN 2008ax (Pastorello et al. 2008; Taubenberger et al. 2011), SN 1993J (Young et al. 1995; Richardson et al. 2006), and SN 2011dh (Bersten et al. 2012), respectively.

From the overall comparison of the SN 2013df light curves in Figures 2 and 3 with the light curves of SNe 1993J and 2011dh, we can make an estimate of the explosion date based, in particular, on when SN 2013df appears to have reached secondary maximum in $B V R I$. We find that all of the light curves indicate that this date is JD 2,456,447.8 \pm 0.5 , or about June 4.3 (indicated in Fig. 2). This is certainly consistent with the earliest discovery epoch of June 7.87 (JD 2,456,451.37). We are unable to constrain the explosion date based on KAIT SN search monitoring, since the observation of the host galaxy with KAIT prior to discovery was on May 25 (i.e., about 10 days before discovery), at a limiting (unfiltered) magnitude of 18.5 .

\subsection{Spectrum}

In Figure 4 we show the Keck/DEIMOS spectrum of SN 2013df and a comparison with spectra of the SNe IIb 1993J (Filippenko et al. 1993), 2008ax (Pastorello et al. 2008; obtained from SUSPECT ${ }^{15}$; the data are also available at WISeREP ${ }^{16}$ ), and 2011dh (available from WISeREP), all at nearly the same age. The SN 1993J explosion date was 1993 March 27.5 (Lewis et al. 1994), so the spectrum of SN 1993J from April 30 shown in the figure is at age $\sim 34 \mathrm{~d}$. The age of the spectrum of SN 2008ax is $\sim 30 \mathrm{~d}$ (Pastorello et al. 2008). The age of the SN 2011dh spectrum is $\sim 28 \mathrm{~d}$ (assuming an explosion date of 2011 May 31.275; Arcavi et al. 2011). From our estimate of the explosion date, above, the SN 2013df spectrum shown in Figure 4 was obtained on day $\sim 37$.

\footnotetext{
15 http://suspect.nhn.ou.edu/ suspect/.

16 Yaron \& Gal-Yam

http://www.weizmann.ac.il/astrophysics/wiserep/.
} 
This spectrum bears a greater similarity with that of SN 1993J than SN 2008ax. Several of the He I lines, notably the $\lambda 6678$ line, atop the broad $\mathrm{H} \alpha$ emission profile, appear to have been stronger in the SN 1993J spectrum (even at a somewhat earlier age) than for SN 2013df. This could imply that the H layer for SN 2013df may have been more substantial (larger mass, larger radius) at explosion, as suggested as well by the post-breakout light-curve behavior of SN 2013df, relative to SN 1993J.

Visible in the spectrum of SN 2013df are absorption features due to $\mathrm{Na}$ I D. In Figure 5 we show these features after the overall continuum in the spectrum has been normalized. One pair of lines appears to be weakly visible at effectively zero redshift, which would correspond to the Galactic foreground extinction contribution; these are indicated as "MW" in the figure. Another pair is far more distinct, at a redshift of 0.002874 , indicated as "Host" in the figure. This is consistent with the redshift for NGC 4414, 0.002388 , given by $\mathrm{NED}^{17}$. We have measured the equivalent width (EW) of each of the $\mathrm{Na}$ I features, $D_{1}$ and $D_{2}$, for each system to be $\operatorname{EW}\left(D_{2}\right)=$ $0.097 \pm 0.011 \AA$ and $\operatorname{EW}\left(D_{1}\right)=0.049 \pm 0.001 \AA$ from the Galactic system, for a total $\mathrm{EW}(\mathrm{Na} \mathrm{I})=0.146 \AA$. For the host-galaxy system, $\mathrm{EW}\left(D_{2}\right)=0.433 \pm 0.002 \AA$ and $\mathrm{EW}\left(D_{1}\right)=0.259 \pm 0.001 \AA$, for a total $\mathrm{EW}(\mathrm{Na} \mathrm{I})=0.691$ $\AA$. The ratio $\operatorname{EW}\left(D_{2}\right) / \operatorname{EW}\left(D_{1}\right)$ is 1.98 for the Galactic component and 1.67 for the host-galaxy component. The ratio of the oscillator strengths of these two lines is 2.0; this intrinsic value is approached only at the lowest optical depths (Munari \& Zwitter 1997). We can see that the $\mathrm{EW}(\mathrm{Na} \mathrm{I})$ [host] is $\sim 4.7$ times that of $\mathrm{EW}(\mathrm{Na} \mathrm{I})$ [Galactic]. If we assume the Galactic foreground extinction estimated by Schlafly \& Finkbeiner (2011; $A_{V}=0.053$ $\mathrm{mag} ;$ ), then from the ratio of these two components, it is plausible that the extinction internal to the host galaxy is $A_{V}=0.25 \mathrm{mag}$.

Poznanski et al. (2011) have stressed that one cannot accurately infer interstellar visual extinction from $\mathrm{EW}(\mathrm{Na} \mathrm{I})$ in low-resolution spectra. The resolution of the Keck DEIMOS spectrum may be just at the limit, however, where we can use the more accurate relations from Poznanski et al. (2012). If we apply their Equation 7 for $\mathrm{EW}\left(D_{2}\right)$, we obtain $E(B-V)=0.11 \pm 0.04$ mag; from their Equation 8 for $\operatorname{EW}\left(D_{1}\right)$, we obtain $E(B-V)=0.08 \pm 0.04 \mathrm{mag}$; and from their Equation 9 for the total $\operatorname{EW}\left(D_{1}+D_{2}\right), E(B-V)=0.09 \pm 0.01$ mag. The uncertainty-weighted mean from all three estimates is then $E(B-V)=0.09 \pm 0.01$ mag. If we assume the Cardelli, Clayton, \& Mathis (1989) reddening law, with $R_{V}=3.1$, then the host-galaxy extinction is $A_{V}=0.28 \pm 0.04 \mathrm{mag}$. (We note that Poznanski et al. 2012 assume the older, somewhat higher values of Galactic extinction from Schlegel et al. 1998.) This estimate of the host-galaxy extinction is quite consistent with the one we made above, which we adopt. We therefore assume hereafter that the total (Galactic foreground plus host galaxy) extinction for SN 2013df is $A_{V}=0.30 \mathrm{mag}$. We adopt an uncertainty in the extinction of \pm 0.05 mag.

\subsection{Progenitor}

17 The NASA/IPAC
http://nedwww.ipac.caltech.edu.

Extragalactic

Database,
We display in Figures $6 \mathrm{a}$ and $6 \mathrm{~b}$ the subregion of the WFPC2 mosaic around the SN position at F555W and F814W, respectively. We show the combination of all the WFC3 exposures in Figure 6c. From a precise comparison between the pre-explosion WFPC2 images and post-explosion WFC3 images, we can identify the SN progenitor. We had previously attempted an identification of the star (Van Dyk et al. 2013a), but this was employing much lower-resolution, ground-based images of the SN. None of the three objects that we had previously nominated as candidates, numbered in Figure 6 , turned out to be the likely SN progenitor. Using 11 stars in common between the WFPC2 and WFC3 images, we astrometrically registered the images to an accuracy of 0.11 drizzled WFPC2 pixel, or 5.5 milliarcsec, and found, instead, that the SN corresponds to the position of the star indicated by the tick marks in Figures $6 \mathrm{a}$ and $6 \mathrm{~b}$. We therefore identify this star as the likely progenitor of SN 2013df.

We extracted photometry for the source from the preexplosion WFPC2 images using Dolphot v2.0 (Dolphin 2000). (We disregarded the short F439W, F606W, and F814W exposures, since the signal-to-noise ratio is quite low in these.) The progenitor location is in the WFPC2 chip 3. The Dolphot output indicates that the progenitor is likely stellar, with an "object type" of 1 ; the "sharpness" parameter, at -0.37 , is slightly outside the acceptable range $(-0.3$ to +0.3$)$ for a "good star" (Dolphin 2000; Leonard et al. 2008). However, if we run Dolphot on the F555W and F814W images only (excluding the F439W images), this parameter has a more acceptable value of -0.25 . We therefore consider it most likely that this is a well-resolved stellar object. The star is not detected by Dolphot in the F439W images (nor is it detectable upon visual inspection of the images). However, we estimate a $3 \sigma$ upper limit to its detection. The Dolphot output automatically includes the transformation from HST flight-system magnitudes to the corresponding Johnson-Cousins magnitudes (Holtzman et al. 1995). We present the Dolphot results for the progenitor in Table 1. We also show the resulting spectral energy distribution (SED) of the star in Figure 7.

To our knowledge, no measurement exists of the metallicity in the vicinity of the SN position (such as from spectroscopy of nearby $\mathrm{H}$ II regions). So, we are unable to quantify accurately the metallicity at the SN site with available data. However, we can provide at least an approximate estimate, based on the assumption that the abundance gradient in the host spiral galaxy follows the same behavior as for the sample of spirals analyzed by Zaritsky et al. (1994). After deprojecting NGC 4414 from its inclination and position angle $\left(56^{\circ}\right.$ and $160^{\circ}$, respectively; Jarrett et al. 2000), we find that SN 2013df occurred $\sim 31^{\prime \prime}$ (or $\sim 2.5 \mathrm{kpc}$ ) from the nucleus. From the Zaritsky et al. nuclear abundance and gradient, then, the oxygen abundance (a proxy for metallicity) at the $\mathrm{SN}$ site is $12+\log [\mathrm{O} / \mathrm{H}] \approx 8.8$, which is comparable to the solar abundance (8.69 \pm 0.05 ; Asplund et al. 2009). Therefore, it is reasonable to assume that the SN site is of roughly solar metallicity.

We compared the observed SED of the progenitor with synthetic SEDs derived from MARCS (Gustafsson et 
al. 2008) supergiant model stellar atmospheres ${ }^{18}$. The model atmospheres are spherical, with standard composition at solar metallicity, surface gravity $\log g=1.0$, and microturbulence of $5 \mathrm{~km} \mathrm{~s}^{-1}$. The spherical-geometry model atmospheres were computed, generally, for stars with masses $0.5,1.0,2.0,5.0$, and $15 M_{\odot}$; we chose models for this last mass as most appropriate for the likely massive progenitor, given what has been inferred for the progenitor initial masses for other $\mathrm{SNe} \mathrm{IIb}$. We found that the synthetic $V-I$ colors, in particular, derived from models at a given temperature are essentially mass-independent (they differ by $\sim 0.03 \mathrm{mag}$ ). The models were reddened based on the assumed total extinction for SN 2013df, above, assuming the Cardelli, Clayton, \& Mathis (1989) reddening law, and were normalized at $V$. The model that provided the best comparison with the photometry has $T_{\text {eff }}=4250 \mathrm{~K}$, which we show in Figure 7. Warmer and cooler models did not compare well with the observations. We therefore adopt this effective temperature for the progenitor, with a generous uncertainty of $\pm 100 \mathrm{~K}$.

The absolute $V$ magnitude of the progenitor, corrected for the assumed distance modulus and extinction to the $\mathrm{SN}$, is $M_{V}^{0}=-6.89 \pm 0.10$. The uncertainty arises from the uncertainties in the photometry, the extinction, and the distance modulus, all added in quadrature. The uncertainty in the transformation from flight-system to Johnson-Cousins magnitudes is $\ll 0.01 \mathrm{mag}$ (Holtzman et al. 1995). The bolometric correction obtained from the MARCS model at $T_{\text {eff }}=4250 \mathrm{~K}$ is $B C_{V}=-0.71$ mag. The uncertainty in $B C_{V}$, resulting from the uncertainty in $T_{\text {eff }}$, is $\sim 0.05$ mag. Including this uncertainty, the bolometric magnitude is $M_{\mathrm{bol}}=-7.60 \pm 0.15$. Assuming a bolometric magnitude for the Sun of 4.74 , this corresponds to a luminosity $L_{\text {bol }}=10^{4.94 \pm 0.06} L_{\odot}$. We show the locus for the progenitor in a HertzsprungRussell (HR) diagram in Figure 8.

\section{DISCUSSION AND CONCLUSIONS}

The SN 2013df progenitor's position in the HR diagram is significantly blueward of the RSG terminus of model massive-star evolutionary tracks with rotation, such as the $15 M_{\odot}$ track (Ekström et al. 2012) in Figure 8. This indicates that the star likely has had its envelope somewhat stripped by some mechanism. The expectation so far for the progenitor scenario for SNe IIb is an interacting massive star binary system, such as for SN 1993J (e.g., Podsiadlowski et al. 1993; Maund et al. 2004) and SN 2011dh (Bersten et al. 2012; Benvenuto et al. 2013). (However, see Crockett et al. 2008 for other possibilities regarding the progenitor of SN 2008ax.) A binary channel is also strongly favored for SNe IIb, in general, based on statistical arguments accounting for their observed relative rate among core-collapse SNe (Smith et al. 2011), as well as on the small ejecta masses and $\mathrm{H} / \mathrm{He}$ line ratios compared to detailed models (Dessart et al. 2011; Hachinger et al. 2012). A blue binary companion is expected to survive the explosion for SNe IIb (Maund et al. 2004; Benvenuto et al. 2013). The locus of the SN 2013df progenitor in the HR diagram is quite similar to, albeit possibly somewhat less luminous than (though within the uncertainties), that of the supergiant progen-

18 http://marcs.astro.uu.se/. itor of SN 1993J (Maund et al. 2004; see also Fig. 8).

We can attempt to assign an initial mass to the progenitor, which, following Van Dyk et al. (2011) for SN 2011dh, would have been $\sim 16-17 M_{\odot}$, based on comparing the locus in $T_{\text {eff }}$ and $L_{\text {bol }}$ to the corresponding values of the 15 and $20 M_{\odot}$ tracks in the figure. On the other hand, adopting the approach of Maund et al. (2011), of basing the likely initial mass on the luminosity at the terminus of the available tracks, we estimate the mass to be $\sim 13-14 M_{\odot}$. As Maund et al. (and references therein) pointed out, the final luminosity at the top of the RSG branch is more relevant to the condition of the yellow supergiant. The timescale for the transition redward for massive stars across the HR diagram is comparatively fast, and the stars will subsequently sit at the RSG locus for the majority of their post-main-sequence evolution. In a binary model, mass transfer will truncate the outer radius of the donor star, resulting in a hotter photosphere; however, the core nuclear-burning luminosity will be relatively unaffected. Note that, if the progenitor experienced mass transfer in a binary, substantial mass stripping can lower the core temperature and, hence, lower the surface luminosity. So, the progenitor could have been somewhat more massive initially, perhaps $\sim 15 M_{\odot}$.

The effective radius of the SN 2013df progenitor $14 \mathrm{yr}$ before explosion, based on our estimates of $T_{\text {eff }}$ and $L_{\mathrm{bol}}$, was $R_{\text {eff }}=545 \pm 65 R_{\odot}$. Unfortunately, we cannot produce an independent estimate from the light curves using the relations between SN progenitor radius and photospheric temperature by Chevalier \& Fransson (2008), Nakar \& Sari (2010), and Rabinak \& Waxman (2011), since these relations tend to break down only a few days after explosion, and the earliest set of photometric data points for SN $2013 \mathrm{df}$ is at age $\sim 10 \mathrm{~d}$. This star was evidently more extended than the progenitor of SN 2011dh - the yellow supergiant progenitor of SN 2011dh modeled by Bersten et al. (2012) has radius $R \approx 200 R_{\odot}$. The later post-shock breakout cooling for SN 2013df implies that its progenitor may have had a larger radius than that of the SN 1993J progenitor. However, both Maund et al. (2004) and Van Dyk et al. (2013b) calculated that the progenitor of SN 1993J had a radius of $\sim 600 R_{\odot}$, which is somewhat greater than, although within the uncertainties of, what we have estimated for the SN 2013df progenitor. In order for the SN 1993J progenitor to have had a smaller effective radius, it must have been hotter $(>4265 \mathrm{~K})$ and of lower luminosity $\left(<10^{5.1} L_{\odot}\right)$ than currently understood (e.g., Maund et al. 2004). To have a larger radius, the SN 2013df progenitor would have had to be cooler and also potentially more luminous. However, to be cooler would require the extinction to be even lower than the small amount that we have estimated. Additionally, the somewhat larger bolometric correction with the lower temperature would be offset by the smaller extinction correction, leading, ultimately, to very little change in the estimated luminosity. At the least, we can say that the SN 2013df and SN 1993J progenitor stars appear to have been relatively comparable in nature.

In summary, we have shown that SN 2013df in NGC 4414 is a SN IIb, based on early-time photometry and spectroscopy, and that its properties are most similar 
to those of SN 1993J. However, the mass of ${ }^{56} \mathrm{Ni}$ synthesized in the explosion likely differed from that in SNe 1993J, 2008ax, and 2011dh. Furthermore, the light curves of SN 2013df - specifically, the late cooling from post-shock-breakout maximum - indicate that its progenitor must have been more extended in radius than that of SN 1993J. Using archival pre-SN, high spatial resolution HST images, together with HST images of SN 2013df, we have identified a star that we consider to be the likely SN progenitor; it had $T_{\text {eff }} \approx 4250 \mathrm{~K}$ and $L_{\mathrm{bol}} \approx 10^{4.94} L_{\odot}$. Ultimately, verification of this identification of the SN 2013df progenitor should occur when the SN itself has greatly faded, as has been done for the SNe IIb 1993J (Maund \& Smartt 2009) and 2011dh (Ergon et al. 2013; Van Dyk et al. 2013b). This can be accomplished with high-quality images obtained with HST. Deep, very late-time HST imaging in the blue or ultraviolet could reveal a putative blue binary companion star at the SN location, although the fact that SN $2013 \mathrm{df}$ is at least a factor of two more distant than both SNe 2011dh and 1993J could make such a detection a more challenging prospect.

We thank the referee for their comments. This work is based in part on observations made with the NASA/ESA Hubble Space Telescope, obtained from the Data Archive at the Space Telescope Science Institute (STScI), which is operated by the Association of Universities for Research in Astronomy (AURA), Inc., under NASA contract NAS5-26555. Some of the data presented herein were obtained at the W. M. Keck Observatory, which is operated as a scientific partnership among the California Institute of Technology, the University of California and NASA; the observatory was made possible by the generous financial support of the W. M. Keck Foundation. KAIT and its ongoing research were made possible by donations from Sun Microsystems, Inc., the HewlettPackard Company, AutoScope Corporation, Lick Observatory, the NSF, the University of California, the Sylvia \& Jim Katzman Foundation, and the TABASGO Foundation. We thank the RATIR instrument team and the staff of the Observatorio Astronómico Nacional on Sierra San Pedro Mártir. RATIR is a collaboration between the University of California, the Universidad Nacional Autonóma de México, NASA Goddard Space Flight Center, and Arizona State University, benefiting from the loan of an H2RG detector from Teledyne Scientific and Imaging. RATIR, the automation of the Harold L. Johnson Telescope of the Observatorio Astronómico Nacional on Sierra San Pedro Mártir, and the operation of both are funded by the partner institutions and through NASA grants NNX09AH71G, NNX09AT02G, NNX10AI27G, and NNX12AE66G, CONACyT grants INFR-2009-01122785, UNAM PAPIIT grant IN113810, and a UC MEXUS-CONACyT grant. Support for this research was provided by NASA through grants GO-12888 and GO13030 from STScI. A.V.F. and his group at UC Berkeley also wish to acknowledge generous support from Gary and Cynthia Bengier, the Richard and Rhoda Goldman Fund, the Christopher R. Redlich Fund, the TABASGO Foundation, and NSF grant AST-1211916. Research by A.G. is supported by the EU/FP7 via ERC grant n 307260, "The Quantum Universe" I-Core program by the Israeli Committee for planning and budgeting, the ISF, GIF, and Minerva grants, and the Kimmel award. S.B. is supported by the Ilan Ramon Fellowship from ISA.

Facilities: HST(WFPC2), HST(WFC3), Keck, KAIT, RATIR.

\section{REFERENCES}

Ahn, C. P., Alexandroff, R., Allende Prieto, C., et al. 2012, ApJS, 203, 21

Aldering, G., Humphreys, R. M., \& Richmond, M. 1994, AJ, 107, 662

Arcavi, I., Gal-Yam, A., Cenko, S. B., et al. 2012, ApJ, 756, L30

Arcavi, I., Gal-Yam, A., Yaron, O., et al. 2011, ApJ, 742, L18

Asplund, M., Grevesse, N., Sauval, A. J., \& Scott, P. 2009, ARA\&A, 47, 481

Benvenuto, O. G., Bersten, M. C., \& Nomoto, K. 2013, ApJ, 762, 74

Bersten, M. C., Benvenuto, O. G., Nomoto, K., et al. 2012, ApJ, 757,31

Bertin, E., \& Arnouts, S. 1996, A\&AS, 117, 393

Butler, N., Klein, C., Fox, O., et al. 2012, Proc. SPIE, 8446, 34

Cardelli, J. A., Clayton, G. C., \& Mathis, J. S. 1989, ApJ, 345, 245

Castelli, F., \& Kurucz, R. L. 2003, Modelling of Stellar Atmospheres, 210, 20P

Cenko, S. B., Clubb, K. I., Zheng, W., Kelly, P. L., et al. 2013, Central Bureau Electronic Telegrams, 3557, 1

Chevalier, R. A., \& Fransson, C. 2008, ApJ, 683, L135

Chevalier, R. A., \& Soderberg, A. M. 2010, ApJ, 711, L40

Chornock, R., Filippenko, A. V., Li, W., et al. 2011, ApJ, 739, 41

Ciabattari, F., Mazzoni, E., Donati, S., et al. 2013, Central Bureau Electronic Telegrams, 3557, 1

Crockett, R. M., Eldridge, J. J., Smartt, S. J., et al. 2008, MNRAS, 391, L5

Dessart, L., Hillier, D. J., Livne, E., et al. 2011, MNRAS, 414, 2985

Dolphin, A. E. 2000, PASP, 112, 1383
Ekström, S., Georgy, C., Eggenberger, P., et al. 2012, A\&A, 537, A146

Ergon, M., Sollerman, J., Fraser, M., et al. 2013, A\&A, in press (arXiv:1305.1851)

Faber, S. M., Phillips, A. C., Kibrick, R. I., et al. 2003,

Proc. SPIE, 4841, 1657

Filippenko, A. V. 1988, AJ, 96, 1941

Filippenko, A. V. 1997, ARAA, 35, 309

Filippenko, A. V., Li, W. D., Treffers, R. R., \& Modjaz, M. 2001, in Small-Telescope Astronomy on Global Scales, ed. W. P. Chen, C. Lemme, \& B. Paczyński (San Francisco, CA: ASP), 121

Filippenko, A. V., Matheson, T., \& Barth, A. J. 1994, AJ, 108, 2220

Filippenko, A. V., Matheson, T., \& Ho, L. C. 1993, ApJ, 415, L103

Fox, O. D., Kutyrev, A. S., Rapchun, D. A., et al. 2012, Proc. SPIE, 8453, 59

Freedman, W. L., Madore, B. F., Gibson, B. K., et al. 2001, ApJ, 553, 47

Gustafsson, B., Edvardsson, B., Eriksson, K., et al. 2008, A\&A, 486, 951

Hachinger, S., Mazzali, P. A., Taubenberger, S., et al. 2012, MNRAS, 422, 70

Hamann, W.-R., \& Gräfener, G. 2004, A\&A, 427, 697

Holtzman, J. A., Burrows, C. J., Casertano, S., et al. 1995, PASP, 107, 1065

Holwerda, B. W., Gonzalez, R. A., Allen, R. J., \& van der Kruit, P. C. 2005, AJ, 129, 1396

Horesh, A., Stockdale, C., Fox, D. B., et al. 2013, MNRAS, 436, 1258 
TABLE 1

HST Photometry of the SN 2013DF Progenitor ${ }^{\mathrm{a}}$

\begin{tabular}{cccccc}
\hline \hline $\begin{array}{c}\mathrm{F} 439 \mathrm{~W}^{\mathrm{b}} \\
(\mathrm{mag})\end{array}$ & $\begin{array}{c}B^{\mathrm{b}} \\
(\mathrm{mag})\end{array}$ & $\begin{array}{c}\mathrm{F} 555 \mathrm{~W} \\
(\mathrm{mag})\end{array}$ & $\begin{array}{c}V \\
(\mathrm{mag})\end{array}$ & $\begin{array}{c}\mathrm{F} 814 \mathrm{~W} \\
(\mathrm{mag})\end{array}$ & $\begin{array}{c}I \\
(\mathrm{mag})\end{array}$ \\
\hline$>25.65$ & $>25.54$ & $24.535(071)$ & 24.515 & $23.144(055)$ & 23.106 \\
\hline
\end{tabular}

a Uncertainties $(1 \sigma)$ are given in parentheses as millimagnitudes.

b $3 \sigma$ upper limit.

Jarrett, T. H., Chester, T., Cutri, R., et al. 2000, AJ, 119, 2498

Leonard, D. C., Gal-Yam, A., Fox, D. B., et al. 2008, PASP, 120, 1259

Lewis, J. R., Walton, N. A., Meikle, W. P. S., et al. 1994, MNRAS, 266, L27

Li, W., Filippenko, A. V., \& Van Dyk, S. D. 2008, Central Bureau Electronic Telegrams, 1306, 1

Li, W., Leaman, J., Chornock, R., et al. 2011, MNRAS, 412, 1441

Matheson, T., Filippenko, A. V., Barth, A. J., et al. 2000, AJ, 120, 1487

Matthews, K., Neugebauer, G., Armus, L., \& Soifer, B. T. 2002, AJ, 123, 753

Maund, J. R., \& Smartt, S. J. 2009, Science, 324, 486

Maund, J. R., Smartt, S. J., Kudritzki, R. P., Podsiadlowski, P., \& Gilmore, G. F. 2004, Nature, 427, 129

Maund, J. R., Fraser, M., Ergon, M., et al. 2011, ApJ, 739, L37

Miller, J. S., \& Stone, R. P. S. 1993, Lick Obs. Tech. Rep. No. 66

Munari, U., \& Zwitter, T. 1997, A\&A, 318, 269

Murphy, J. W., Jennings, Z. G., Williams, B., Dalcanton, J. J., \& Dolphin, A. E. 2011, ApJ, 742, L4

Nakar, E., \& Sari, R. 2010, ApJ, 725, 904

Pastorello, A., Kasliwal, M. M., Crockett, R. M., et al. 2008, MNRAS, 389, 955

Perets, H. B., Gal-Yam, A., Mazzali, P. A., et al. 2010, Nature, 465, 322

Podsiadlowski, P., Hsu, J. J. L., Joss, P. C., \& Ross, R. R. 1993, Nature, 364, 509

Poznanski, D., Ganeshalingam, M., Silverman, J. M., \& Filippenko, A. V. 2011, MNRAS, 415, L81

Poznanski, D., Prochaska, J. X., \& Bloom, J. S. 2012, MNRAS, 426,1465

Rabinak, I., \& Waxman, E. 2011, ApJ, 728, 63 (Erratum: 2013, ApJ, 770, 81)

Richardson, D., Branch, D., \& Baron, E. 2006, AJ, 131, 2233

Richmond, M. W., Treffers, R. R., Filippenko, A. V., et al. 1994, AJ, 107, 1022

Richmond, M. W., Treffers, R. R., Filippenko, A. V., \& Paik, Y. 1996, AJ, 112, 732

Roming, P. W. A., Pritchard, T. A., Brown, P. J., et al. 2009, ApJ, 704, L118
Ryder, S. D., Murrowood, C. E., \& Stathakis, R. A. 2006, MNRAS, 369, L32

Schaefer, B. E. 1998, ApJ, 509, 80

Schlafly, E. F., \& Finkbeiner, D. P. 2011, ApJ, 737, 103

Schlegel, D. J., Finkbeiner, D. P., \& Davis, M. 1998, ApJ, 500, 525

Shivvers, I., Mazzali, P., Silverman, J. M., et al. 2013, MNRAS, 436, 3614

Silverman, J. M., Mazzali, P., Chornock, R., Filippenko, A. V., Clocchiatti, A., Phillips, M. M., Ganeshalingam, M., \& Foley, R. J. 2009, PASP, 121, 689

Skrutskie, M. F., Cutri, R. M., Stiening, R., et al. 2006, AJ, 131, 1163

Smith, N., Li, W., Filippenko, A. V., \& Chornock, R. 2011, MNRAS, 412, 1522

Stancliffe, R. J., \& Eldridge, J. J. 2009, MNRAS, 396, 1699

Stetson, P. B. 1987, PASP, 99, 191

Taubenberger, S., Navasardyan, H., Maurer, J. I., et al. 2011, MNRAS, 413, 2140

Thornley, M. D., \& Mundy, L. G. 1997, ApJ, 490, 682

Vallejo, O., Braine, J., \& Baudry, A. 2002, A\&A, 387, 429

Van Dyk, S. D., Cenko, S. B., Foley, R. J., et al. 2013a, The Astronomer's Telegram, 5139, 1

Van Dyk, S. D., Garnavich, P. M., Filippenko, A. V., et al. 2002, PASP, 114, 1322

Van Dyk, S. D., Li, W., Cenko, S. B., et al. 2011, ApJ, 741, L28

Van Dyk, S. D., Zheng, W., Clubb, K. I., et al. 2013b, ApJ, 772, L32

Watson, A. M., Richer, M. G., Bloom, J. S., et al. 2012,

Proc. SPIE, 8444,5

Woosley, S. E., Eastman, R. G., Weaver, T. A., \& Pinto, P. A. 1994, ApJ, 429, 300

Woosley, S. E., Heger, A., \& Weaver, T. A. 2002, Reviews of Modern Physics, 74, 1015

Yaron, O., \& Gal-Yam, A. 2012, PASP, 124, 668

Young, T. R., Baron, E., \& Branch, D. 1995, ApJ, 449, L51

Zaritsky, D., Kennicutt, R. C., Jr., \& Huchra, J. P. 1994, ApJ, 420, 87 


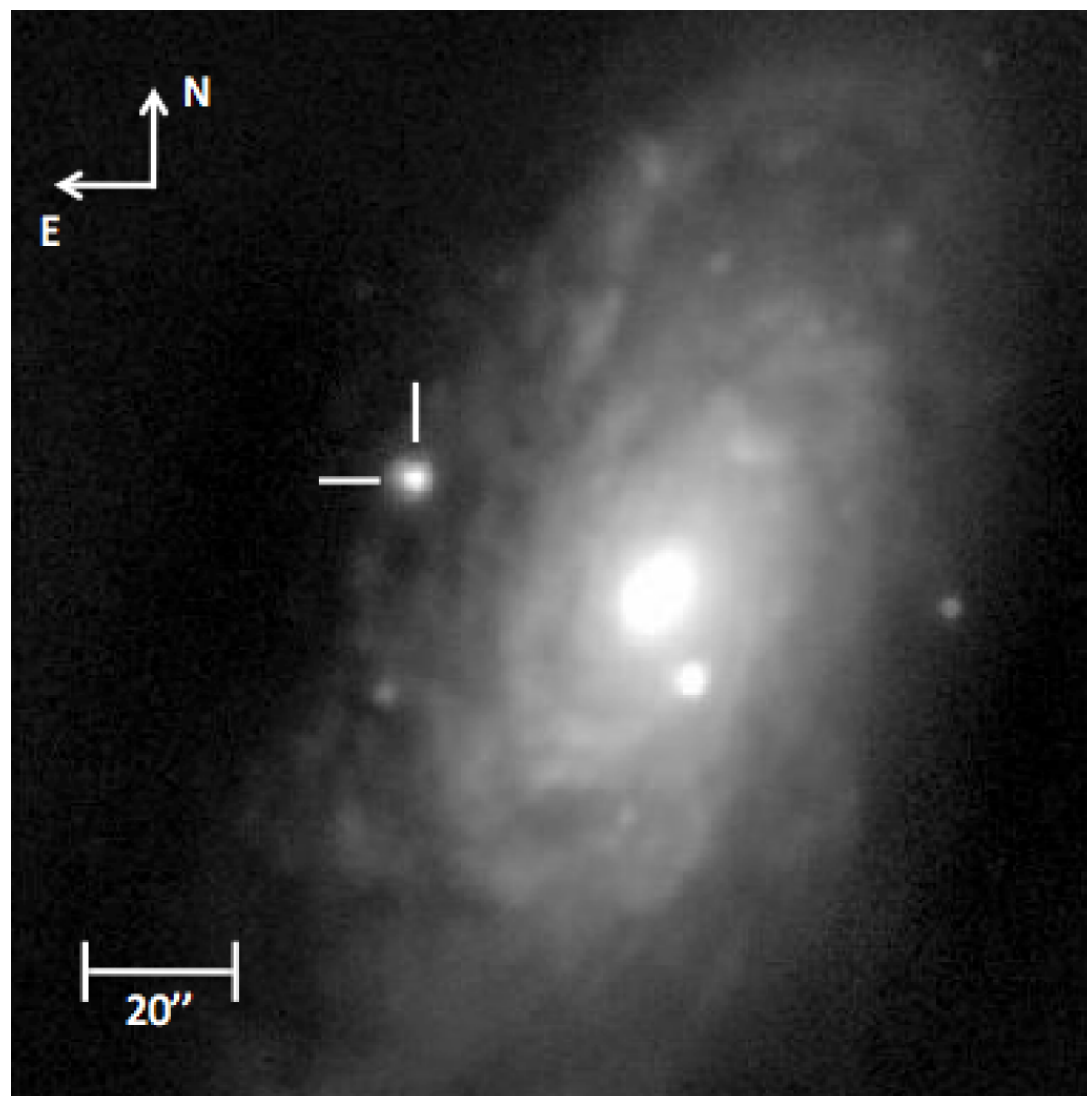

FIG. 1.- A greyscale composite of rizJH images obtained with RATIR on 2013 June 24, showing SN 2013df in NGC 4414. The position of the $\mathrm{SN}$ is indicated by the tick marks. North is up, east is to the left. 


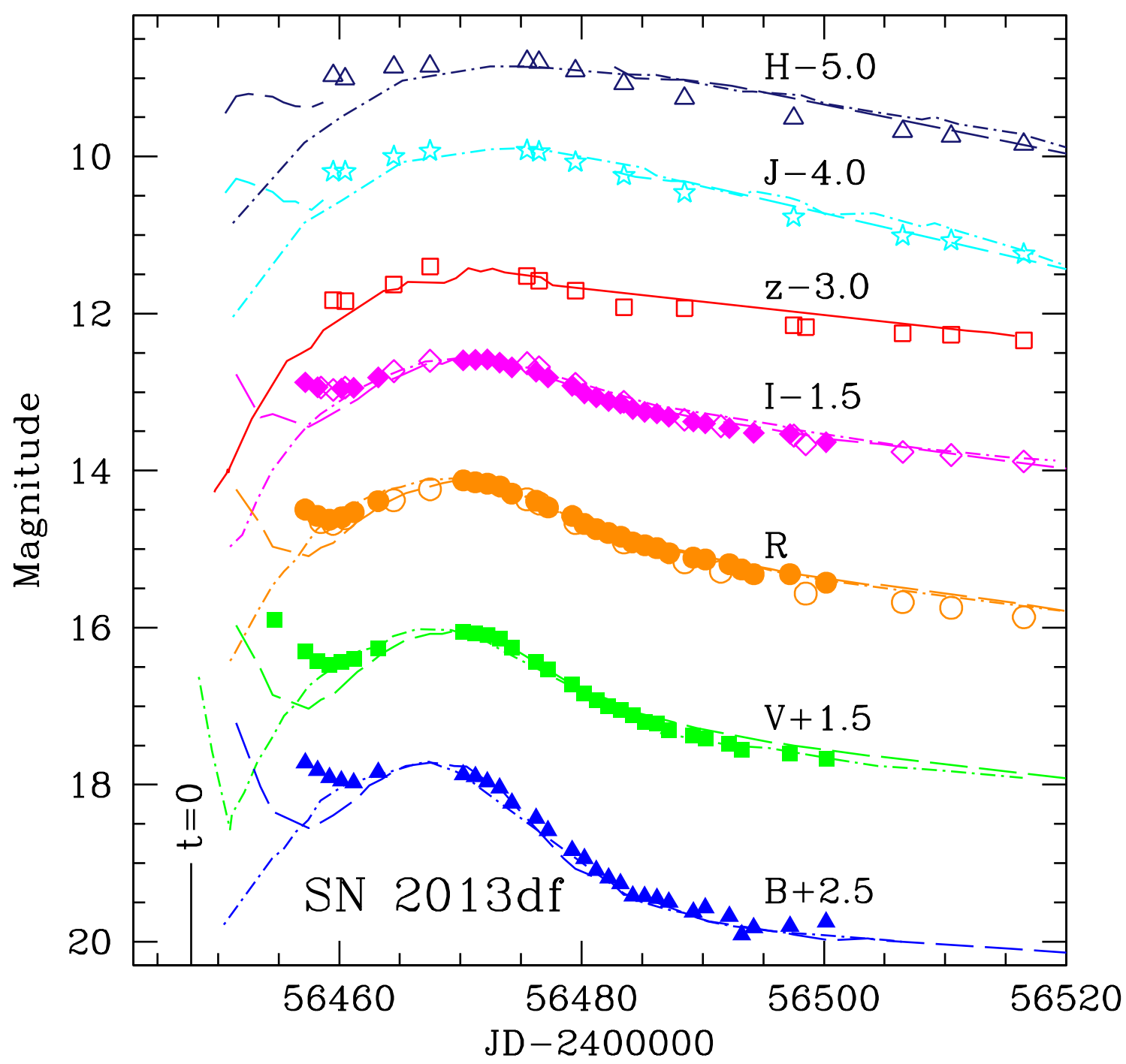

FIG. 2.- BVRIzJH light curves of SN 2013df from KAIT (solid points) and RATIR (open points; with $r$ and $i$ converted to $R$ and $I$, respectively, following http://www.sdss.org/dr7/algorithms/sdssUBVRITransform.html\#Lupton2005). The observed curves have been offset from each other for clarity. Shown for comparison are the BVRIJH light curves of SN 1993J (Richmond et al. 1994; 1996; Matthews et al. 2002; dashed lines) and of SN 2011dh (Arcavi et al. 2011; Van Dyk et al. 2013b; Ergon et al. 2013; dot-dashed lines), and the $z$-band light curve of SN 2008ax (Pastorello et al. 2008; solid line), all adjusted in time and relative brightness. The estimated time $t=0$ of the explosion is indicated. 


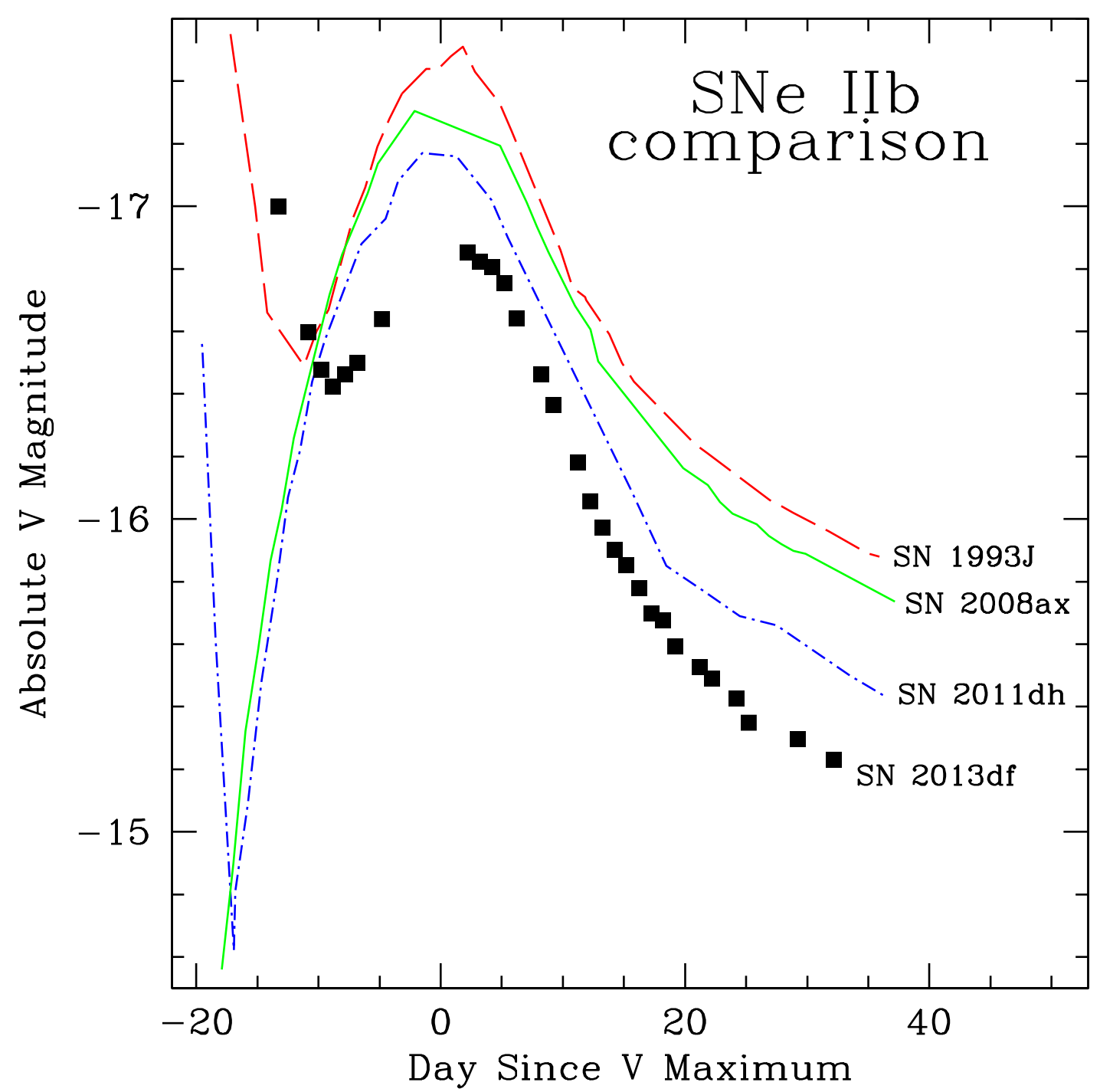

FIG. 3.- Absolute $V$ light curve for SN 2013df (solid points), compared to those at $V$ for SNe 1993J (dashed line; colored red in the online version), 2008ax (solid line; colored green in the online version), and 2011dh (dot-dashed line; colored blue in the online version) shown in Figure 2. All of the curves are displayed relative to the day of $V$ maximum; see text. 


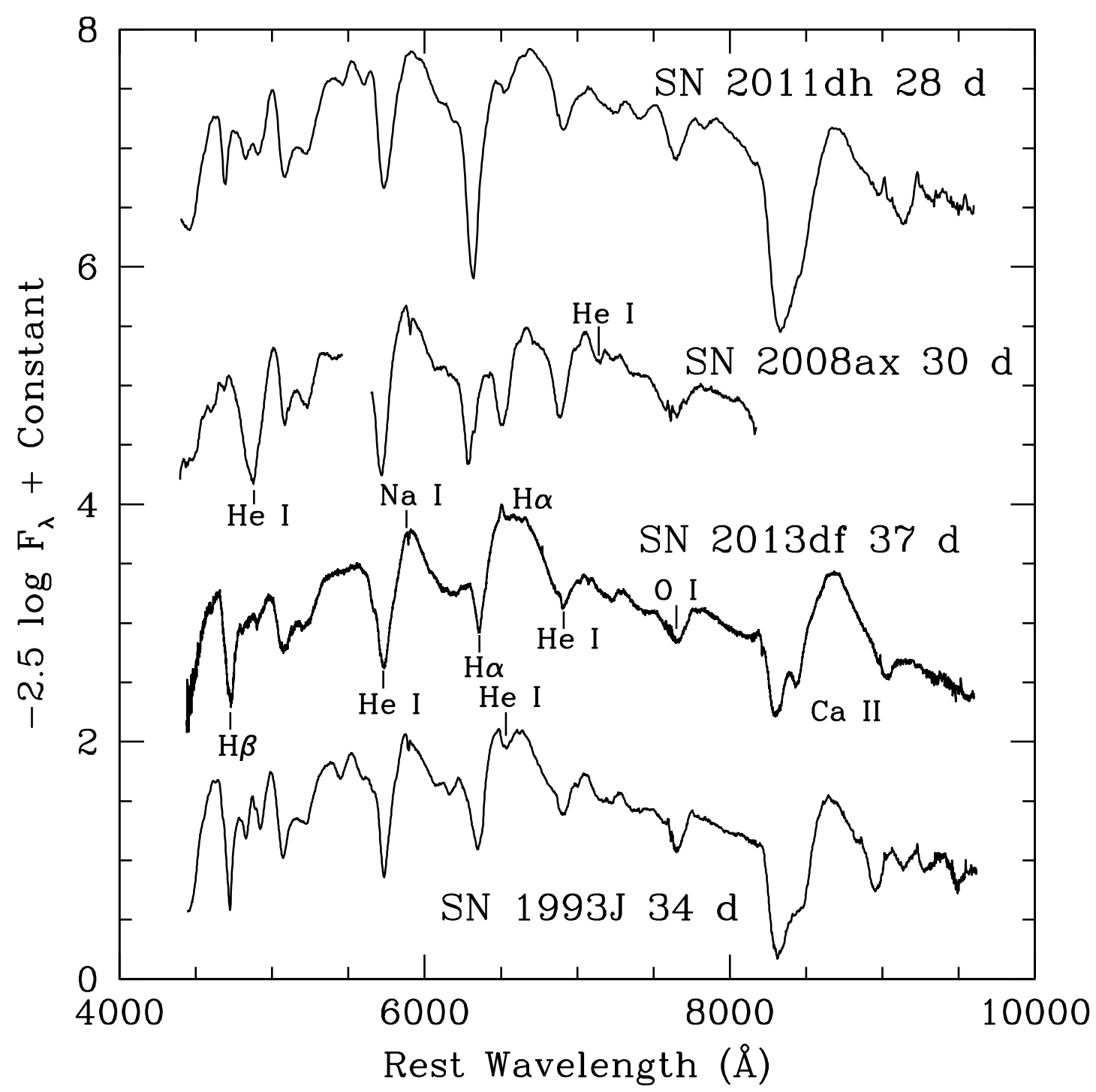

FIG. 4.- Optical spectrum of SN 2013df obtained on 2013 July 11.2 with DEIMOS on the Keck II 10-m telescope. Also shown for comparison are the spectra, at approximately the same age, of SN 1993J from 1993 April 30 (Filippenko et al. 1993), SN 2008ax from 2008 April 2 (Pastorello et al. 2008), and SN 2011dh from 2011 June 29 (unpublished; from WISeREP, Yaron \& Gal-Yam 2012). Several spectral features are indicated. 


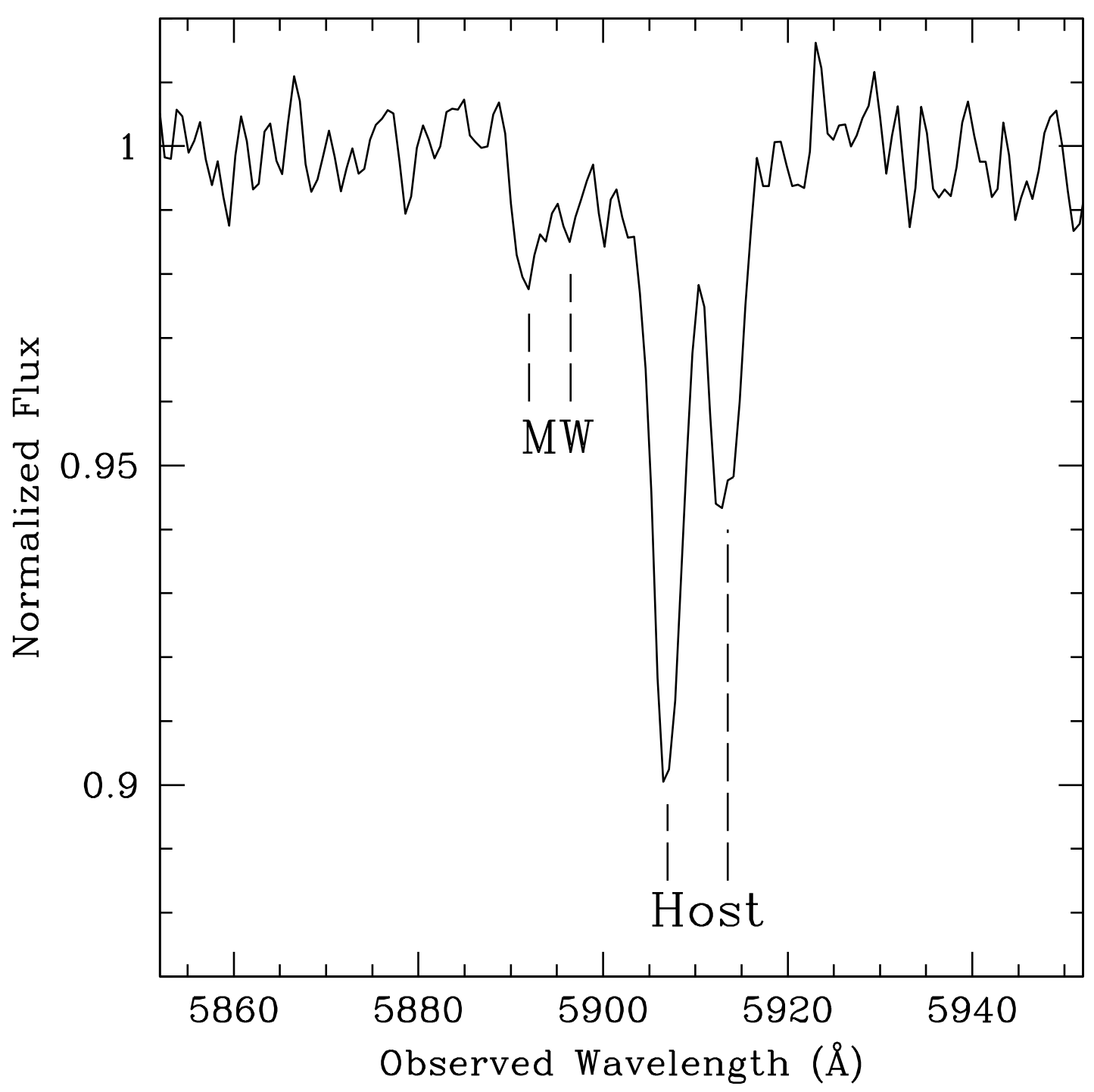

FIG. 5.- Portion of the spectrum of SN 2013df shown in Figure 4, after normalization of the continuum, focusing on the region including the interstellar Na I D $\lambda \lambda 5890.0,5895.9$ absorption features. The possible feature associated with the Galactic component is indicated by the dashed lines as "MW," and the feature (corresponding to a redshift of 0.002874) associated with the host galaxy is indicated as "Host." 


\section{SN $2013 d f$ \\ (a) 1999 Apr 29}

F555W

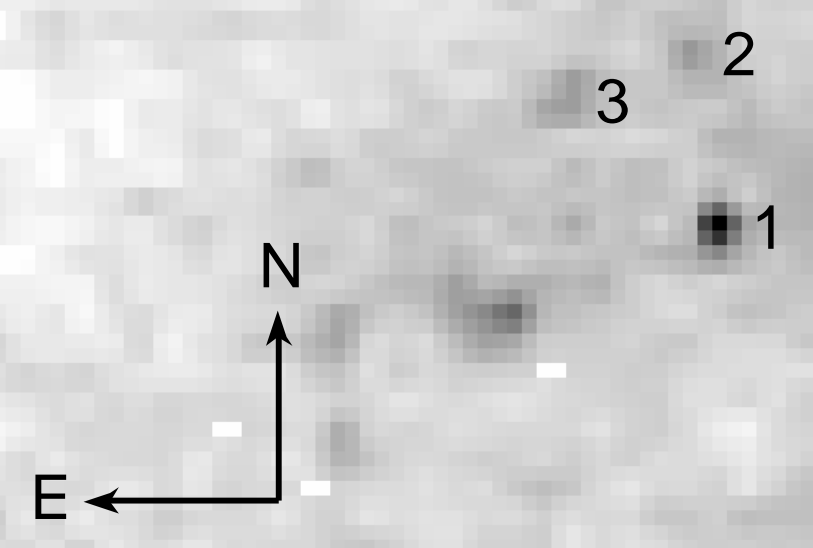

FIG. 6.- A portion of the archival HST WFPC2 images of NGC 4414 from 1999 in (a) F555W and (b) F814W. The likely progenitor of SN 2013df is indicated by tick marks. (c) A portion of the HST WFC3 F555W image of SN 2013df, to the same scale and orientation. The SN is indicated by tick marks. We have astrometrically registered the WFPC2 and WFC3 images to an accuracy of 5.5 milliarcsec. The three progenitor candidates initially identified by Van Dyk et al. (2013a) are indicated ("1," "2," "3") in panel (a). North is up, east is to the left. 
SN 2013df

(b) 1999 Apr 29

F814W
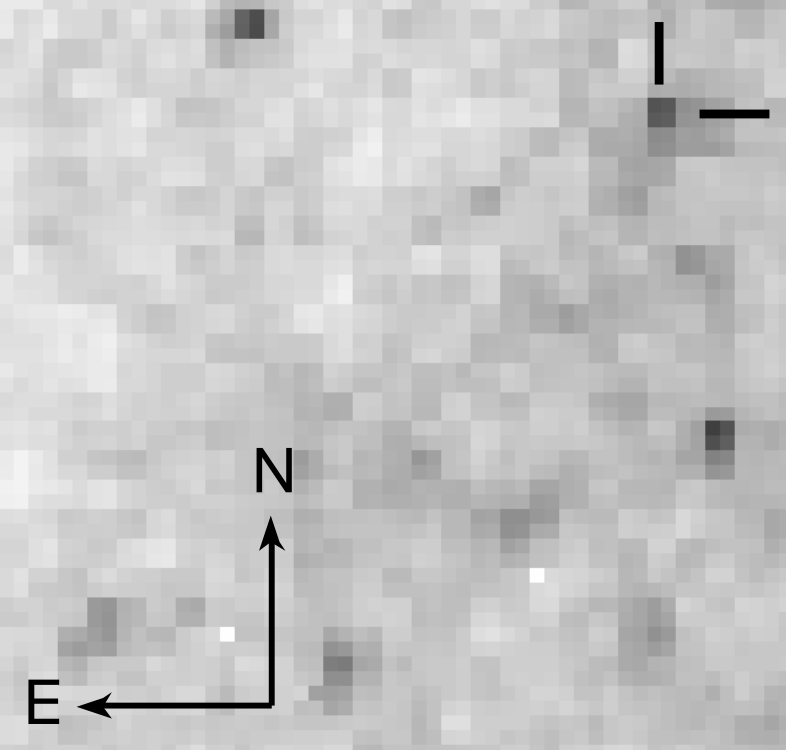

FIG. 6.- (Continued.) 


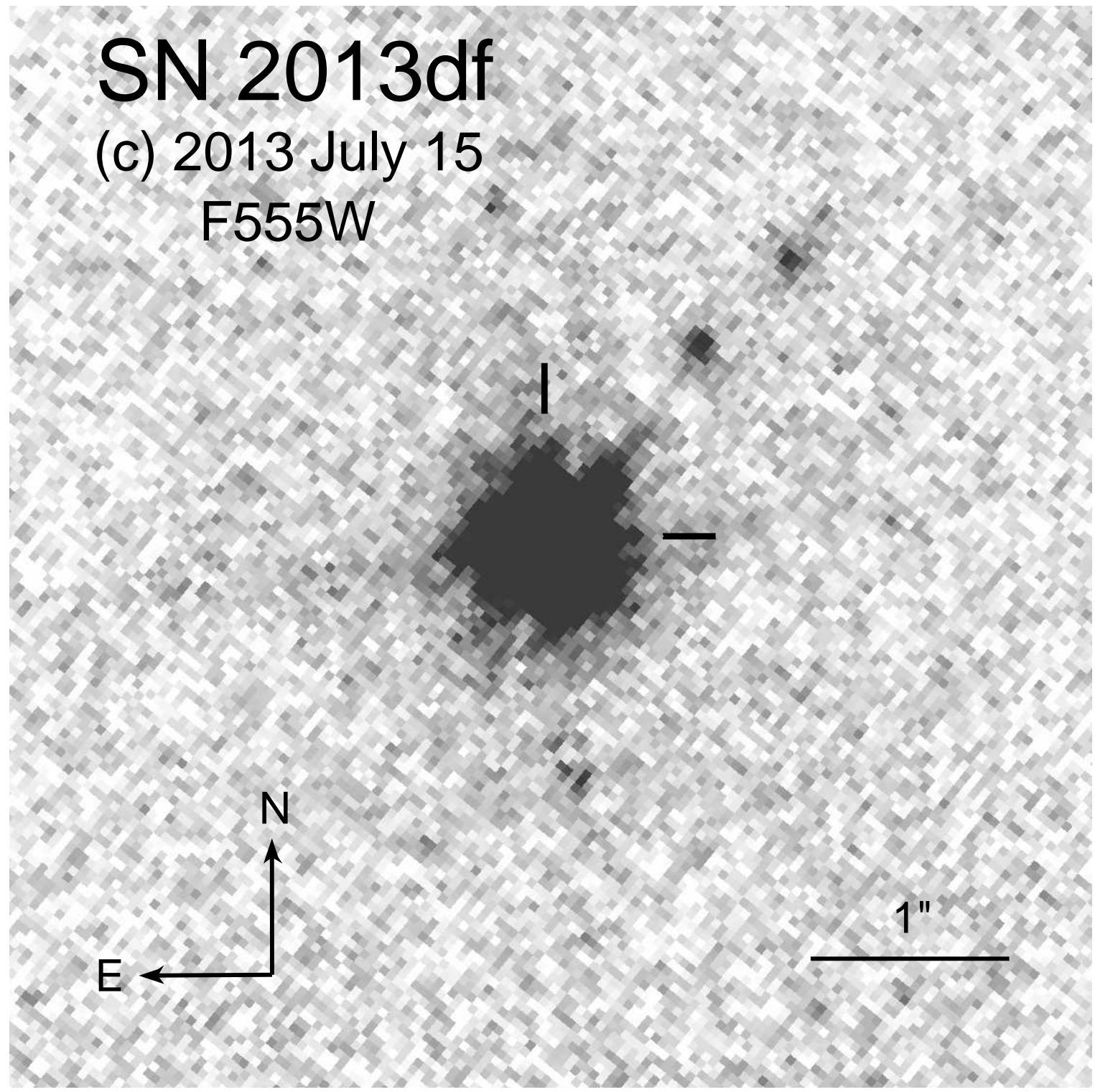

FIG. 6.- (Continued.) 


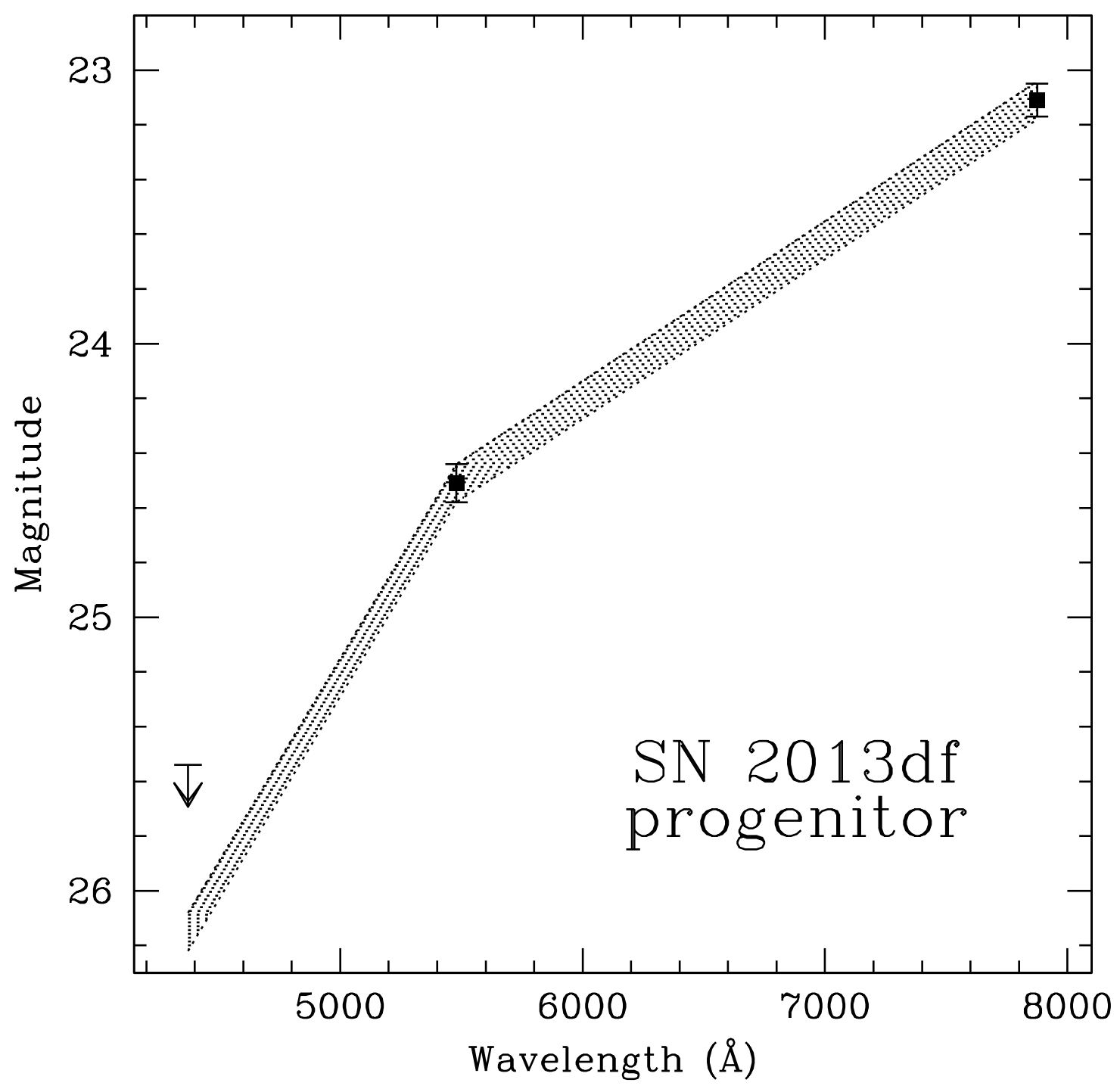

FIG. 7.- Spectral energy distribution of the probable progenitor star of SN 2013df, based on the photometry presented in Table 1. Shown for comparison is synthetic photometry derived from a MARCS (Gustafsson et al. 2008) supergiant model atmosphere at solar metallicity with effective temperature $4250 \mathrm{~K}$, reddened assuming $A_{V}=0.30 \mathrm{mag}$ and the Cardelli, Clayton, \& Mathis (1989) reddening law. The model has been normalized at $V$. The hashed region in the diagram represents the range in brightness for the model within the total uncertainty in the observed $V$ magnitude. 


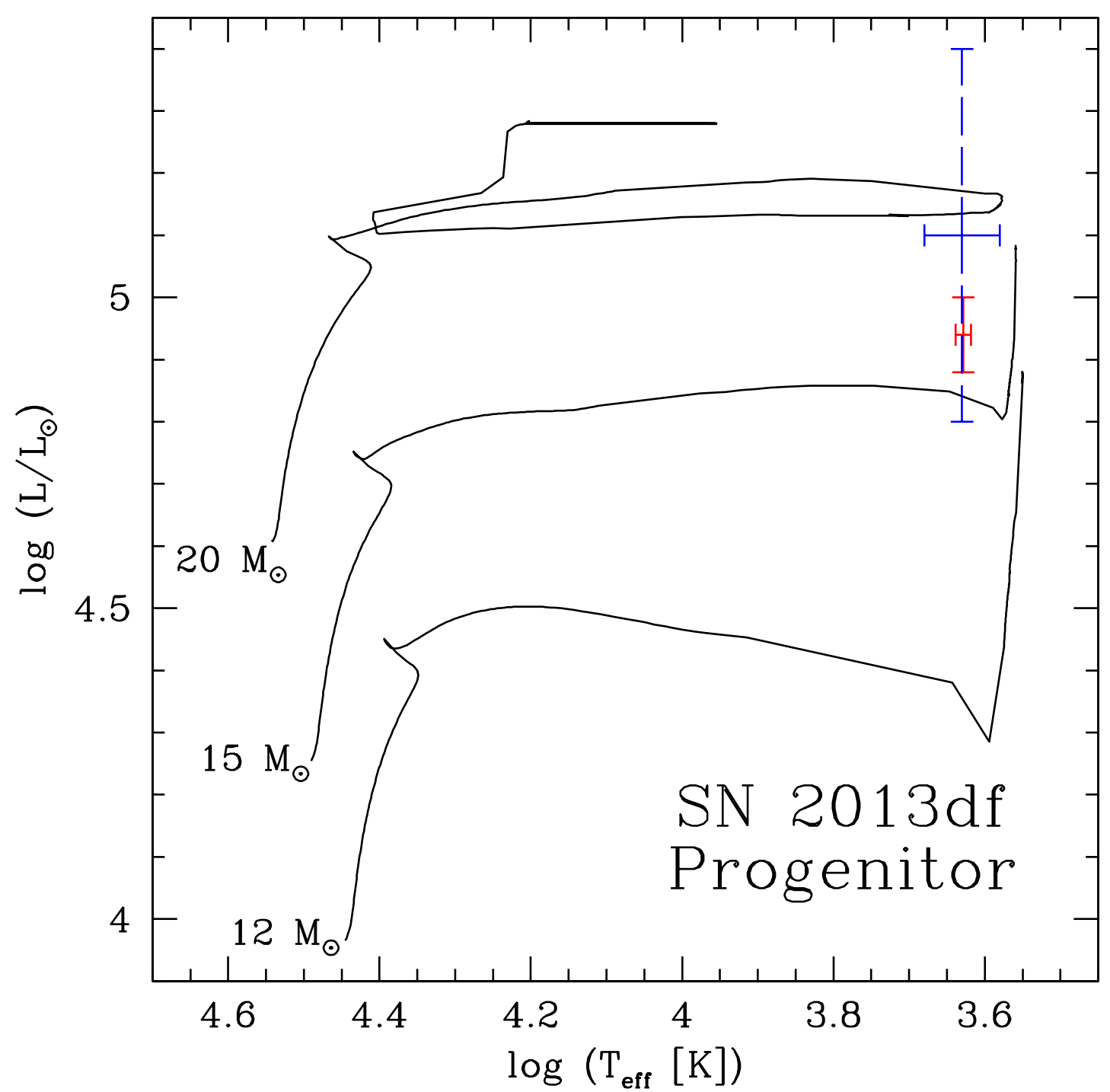

FIG. 8.- Hertzsprung-Russell diagram, showing the locus of the probable progenitor of SN 2013df (solid symbol; colored red in the online version). For comparison we also illustrate the massive-star evolutionary tracks with rotation from Ekström et al. (2012) at initial masses 12,15 , and $20 \mathrm{M}_{\odot}$ (curves), as well as the locus of the SN 1993J progenitor (dashed symbol; colored blue in the online version) from Maund et al. (2004). 